\title{
La gestión de servicios de la Sucursal Ventas Internacionales de la agencia de viajes Gaviota Tours con los tour operadores alemanes Aventoura Cubareisen y TourCom.
}

The service management of the International Sales Branch of the travel agency Gaviota Tours with the German tour operators Aventoura Cubareisen and TourCom.

MSc. Maité Rodríguez González ${ }^{1}$, Lic. Claudia Viamonte Olivera ${ }^{2}$, Est. Yoan Hernández Flores $^{3}$, Yennifer Sánchez Borges. ${ }^{4}$ \& DrC. Efraín Velasteguí López ${ }^{5}$

Recibido: 10-11-2018 / Revisado: 17-02-2019 /Aceptado: 25-02-2019/ Publicado: 05-04-2019

\begin{abstract}
.
DOI: https://doi.org/10.33262/cienciadigital.v3i1.309

The management of services is a fundamental element by which the direction of all the companies destined to tourism must be guided. Precisely travel agencies are a key element of these entities in this sector, and, because of the importance they have, it is necessary to study to diagnose the management of their services. Therefore, the present investigation aims to: Value the management of services in the travel agency Gaviota Tours International Sales with the German tour operators Aventoura Cubareisen and TourCom; for which a methodology was used that allowed the analysis of this topic. Different methods were used, such as theoretical, empirical and statistical-mathematical, through the application of techniques such as interviews with specialists and managers of the agency and tour operators, surveys of external clients, the Pareto diagram and the diagram of affinity. The results obtained showed the main services, factors and gaps between the different levels of service that influence the decrease in revenues by the German market, allowing to identify the main deficiencies of the agency when it comes to managing its services with Aventoura Cubareisen and TourCom, all of which allowed for a proposal of
\end{abstract}

\footnotetext{
${ }^{1}$ Universidad de la Habana, Facultad de Turismo, La Habana, Cuba, mrodriguez@ftur.uh.cu ${ }^{2}$ Universidad de la Habana, Facultad de Turismo, La Habana, Cuba, ybencomo@ftur.uh.cu ${ }^{3}$ Universidad de la Habana, Facultad de Turismo, La Habana, Cuba, yoan_flores@estudiantes.ftur.uh.cu ${ }^{4}$ Universidad de la Habana, Facultad de Turismo, La Habana, Cuba, yennifer_bg@estudiantes.ftur.uh.cu ${ }^{5}$ Ciencia Digital, Ambato Ecuador, luisefrainvelastegui@cienciadigital.org
} 
improvement actions to eradicate said problems in order to increase customer satisfaction.

Keywords: Service management, tourism, tour operators

\section{Resumen.}

La gestión de servicios es un elemento fundamental por el que debe guiarse la dirección de todas las empresas destinadas al turismo. Precisamente las agencias de viajes son un elemento clave de estas entidades en dicho sector, y, por la importancia que poseen, se hace necesario el estudio dirigido a diagnosticar la gestión de sus servicios. Por ello la presente investigación tiene como objetivo: Valorar la gestión de servicios en la agencia de viajes Gaviota Tours Ventas Internacionales con los tours operadores alemanes Aventoura Cubareisen y TourCom; para lo cual se empleó una metodología que permitió el análisis de dicho tema. Se utilizaron diferentes métodos como el teórico, empírico y el estadístico-matemático, a través de la aplicación de técnicas como la entrevistas a especialistas y directivos de la agencia y de los tours operadores, encuestas a clientes externos, el diagrama de Pareto y el diagrama de afinidad. Los resultados obtenidos arrojaron los principales servicios, factores y brechas existentes entre los diferentes niveles de servicio que influyen en la disminución de los ingresos por parte del mercado alemán, permitiendo así identificar las principales deficiencias de la agencia a la hora de gestionar sus servicios con Aventoura Cubareisen y TourCom, todo lo cual permitió realizar una propuesta de acciones de mejora para erradicar dichos problemas en aras de aumentar la satisfacción de los clientes.

Palabras claves: Gestión de servicios, turismo, tour operadores

\section{Introducción}

Entre las agencias de viajes del destino Cuba se destaca Gaviota Tours, perteneciente al grupo de turismo Gaviota S.A. La misma aspira a un crecimiento con tour operadores que prestigien su actividad, y a los cuales pretende darle un servicio personalizado. Dicha agencia cuenta con una Casa Matriz y seis sucursales de ventas territoriales. Situada en la Casa Matriz se encuentra la Sucursal Gaviota Tours Ventas, creada en el año 2015, cuyo objetivo fundamental son las ventas internacionales. Actualmente el mercado que más ingresos y número de pax le aporta a la agencia es el europeo. En el primer trimestre del año 2017, el mercado Europa mostró un crecimiento de 5455 pax y elevó sus ingresos con relación al primer trimestre del año 2016. Al comparar dichos datos con respecto al primer trimestre del año 2018, el mercado también mostró un crecimiento acelerado del número de pax. Los países que más crecimiento mostraron en ese mercado fueron Alemania y Francia. El análisis del incremento en ambos emisores, concluyó que con respecto al 2016 Alemania tuvo un 
aumento de 1128 pax, mostrando una disminución de los ingresos en 7273.31 USD, mientras que, comparando el mismo trimestre del 2018 con respecto a los dos años anteriores, también se evidencia un aumento del número de pax, pero una disminución del $5 \%$ en los ingresos. Sin embargo, Francia tuvo un crecimiento en el 2017 de 4173 pax y un aumento de los ingresos de aproximadamente 666.548 USD, lo mismo ocurre con el año 2018, el cual mostró un aumento del número de pax y un crecimiento en sus ingresos, con respecto al mismo período de los dos años anteriores. Aunque Alemania ha mostrado un descenso de los ingresos a la agencia, dicho país y Francia siguen siendo los que más ganancias le generan a la entidad, así como los que mayor número de visitantes aportan.

La mayoría de los servicios como el de alojamiento, servicio de autos, paquetes turísticos o transporte terrestre, muestran una disminución de ingresos para la agencia.

Por ello realizar una valoración de la gestión de servicios en la agencia de viajes Gaviota Tours Ventas Internacionales con los tours operadores alemanes Aventoura Cubareisen y TourCom permitirá establecer acciones, las cuáles eleven la calidad del servicio en la entidad y con ello lograr un mayor índice de satisfacción de los clientes.

Para comprender las terminologías agencias de viajes, gestión y servicios, se consultaron un conjunto de autores especialistas en las conceptualizaciones anteriores. Al analizar los conceptos brindados por autores como Villamayor y Lamas (1998), Benavides (2011) y Ulacia, (2015), se puede definir la gestión de servicios turísticos como aquellas acciones llevadas a cabo para alcanzar los objetivos propuestos por la empresa, por medio, de la planeación, organización, dirección y control de las entidades que interactúan con los clientes, prestando sus diferentes servicios para satisfacer las necesidades de los mismos. Con respecto a las definiciones de agencias de viajes, se pudo comprobar que diferentes autores como Fernández y Blanco (1996) y Saldaña (2013) han conceptualizado de diversas formas estas empresas de servicios, coincidiendo en definirlas como una empresa mercantil, las cuales tiene como propósito brindar al cliente servicios relacionados con el turismo, por medio de sus diferentes funciones: la intermediación, el asesoramiento y la organización, jugando un rol mediador entre la persona que demanda el servicio y el productor de esos servicios turísticos.

En la actualidad es primordial que las empresas turísticas, deben tengan implementado un sistema de servicio, en el cual converjan cuatro subsistemas fundamentales: el gerencial, donde se toman las principales decisiones del negocio; el de reglas y normas, que lo conforman las regulaciones de la organización; el técnico, que está conformado por las herramientas y técnicas utilizadas para realizar el producto servicio; y el social que refiere al sistema humano existente en la entidad (Ulacia, 2015). Para desarrollar un sistema de gestión de servicio, en coherencia con las necesidades de los clientes, hay que tener presente diferentes aspectos, en los cuales debe centrarse la empresa a la hora de brindar sus diferentes servicios. Según Ulacia, (2007) estos serían: valor del cliente, estrategia del servicio, misión- 
visión del servicio, sentido de pertenencia, orientación al cliente, trabajo en equipo, intangibilidad, heterogeneidad y la inseparabilidad.

En la actualidad el uso de las Tecnologías de la Información y las Comunicaciones (TICs) es una de las herramientas que resulta imprescindible implementar para lograr una óptima gestión de los servicios en empresas turísticas y fundamentalmente en las agencias de viajes. Para una agencia de viajes, es imprescindible el uso del Internet, pues ofrece una serie de beneficios, como son, la reducción de costos, disponibilidad de tiempos y una mayor cobertura del mercado, lo que conlleva, al aumento de la rentabilidad de la entidad. Además, permite una retroalimentación más eficiente entre la agencia y sus clientes, debido a que los especialistas de marketing en-línea, pueden comunicarse con sus consumidores y conocer sus criterios en el momento. Entre las principales ventajas de una agencia de viajes on-line está, la identificación de nuevos nichos de mercados, permitiendo, conocer los principales deseos de los clientes, a través de una mejor administración de la información, lo que posibilita, que la oferta de productos/servicios esté acorde con las expectativas y permita una mejor comercialización de sus paquetes turísticos (Castaño y Castillo, 2015).

\section{Metodología utilizada}

Para el desarrollo de la investigación, se utilizaron métodos teóricos como el hipotéticodeductivo; el analítico- sintético y el histórico- lógico; métodos empíricos como la observación, entrevistas en profundidad a especialistas y encuestas; y método estadísticomatemático, el cual permitió el análisis y la tabulación de las encuestas se llevó a cabo el procesador SSPS versión 22 y el Software Microsoft Excel.

Es imprescindible además que, para lograr una gestión eficaz de los servicios, se hace necesario el estudio de varias herramientas que garanticen a la entidad su diferenciación en el sector. Entre las herramientas estudiadas sobresalen diferentes modelos utilizados para la gestión de servicios, los que brindan una ventaja para la organización, ya que, en poco tiempo, pueden existir mejoras significativas, tanto para las empresas, como para sus clientes. Dentro de los modelos fundamentales se encuentran el modelo de Carlzon (1991), Arana y Ginebra (1991), citado en Fernández, C. y Blanco, A (1996) y el de Fisher, (1994).

A través del análisis de los modelos anteriores, se concluye que en ellos hay un aspecto en común, el cual se debe tener en cuenta a la hora de establecer un modelo de gestión de servicios, y es precisamente que en los tres modelos se señala la importancia de conocer las necesidades y expectativas de los clientes externos. Además, se evidencia el proceso de retroalimentación que debe existir entre los clientes y la entidad, para poder lograr una gestión de servicios que diferencie a la organización de otras entidades que, tal vez, brinden un servicio similar, logrando así la fidelización de los clientes. 
La propuesta de una metodología de gestión de servicios para llevar a cabo por parte de la agencia con los tour operadores alemanes, se deriva del análisis de las metodologías de Carlzon (1991) y Fisher (1994), pues son las más enfocadas al tema de gestión de servicios, y muestran claramente en sus pasos lo que deben realizar las organizaciones para brindarle a sus clientes un servicio de calidad, y trazar acciones de mejoras en las entidades. Se tuvo en cuenta además para definir la metodología que a continuación se explica, los criterios de la Dr. C Zoila Ulacia Oviedo, especialista en el tema estudiado.

La metodología consta de 7 fases, los cuales están enfocados a actividades relacionadas con la planificación, la organización, el control y la dirección de los servicios de la agencia de viajes.

A pesar de que esta metodología cuenta con 7 fases, la investigación se centrará en los tres primeros de la misma, ya que son los que dan cumplimiento al objetivo de la investigación.

Fase I: Auditoría del servicio: Este paso se basa en conocer qué están pensando los clientes, cuáles son sus necesidades, deseos y expectativas, a través de una verificación general de los puntos de contactos, estos puntos demuestran la interacción cliente-entidad. Esta primera fase se desarrollará en 3 etapas fundamentales:

Etapa I: Disponer de una serie válidas de criterios para la calidad del servicio: La garantía de una auditoría de servicio confiable se centra en la definición de criterios necesarios de calidad del servicio. La formación de estos criterios requiere comprender de manera exacta, lo que los clientes necesitan y desean. Esta es una información con la cual se cuenta, de haber realizado un proceso de retroalimentación efectivo. Es en esta primera fase donde se manifiesta el control que debe existir sobre los requerimientos de los clientes, ya que se entiende por control; al proceso que asegura que lo real se corresponde con lo proyectado, además de ser un proceso de monitorear, comparar y corregir el desempeño laboral (Porteando, 2015).

Para la realización de esta primera etapa se identificaron los atributos básicos de los servicios, a través de una tormenta de ideas, realizada a un grupo de 5 especialistas del tour operadores, estos fueron mencionados con anterioridad. Esto permitió la determinación de los atributos claves del éxito, y, posteriormente, se compararon los criterios de la agencia con respecto a los emitidos por los tours operadores, identificando los atributos que deben ser mejorados y el establecimiento de los estándares de realización. Finalmente, se aplicó una encuesta a los directivos comerciales y especialistas de calidad, con el objetivo de diseñar la matriz de actitud personal.

Etapa II: Estudiar los ciclos fundamentales que tienen los clientes: el estudio de los ciclos de servicio por los que pasa el cliente, permite identificar los momentos de verdad incluidos en cada uno de ellos. Para la realización de esta etapa se utilizó como herramienta la observación, puesto que permitió definir los principales ciclos de servicio de la agencia con 
los tours operadores, y luego poder identificar los momentos críticos de verdad por los que atraviesa la entidad durante un proceso de gestión de servicios. Sobresalieron como ciclos fundamentales, la interacción tour operador-agencia y la compra del programa.

Etapa III: Identificación de las brechas entre las expectativas del cliente y el servicio: una vez identificados los criterios de calidad definidos, los ciclos de servicios y los momentos críticos de verdad asociados a estos, el siguiente paso consistió en el procesamiento de la información, lo que permitió detectar los principales momentos y áreas de la agencia que están ocasionando problemas, todo lo cual facilitó la determinación de los factores fundamentales que influyen en la gestión de los servicios en la agencia.

Para la identificación de estas brechas, se tomó como referencia la relación que existe entre los beneficios que brinda la agencia, y lo que percibe el cliente. Fueron determinadas mediante entrevistas realizadas a los especialistas comerciales del tour operadores y de la aplicación de la técnica Tormenta de ideas. Estos análisis permitieron definir las brechas existentes entre el servicio prometido, servicio ofrecido, servicio entregado y el servicio percibido. Además, se aplicaron encuestas a clientes externos, para la determinación de un criterio más general, y entrevistas a directivos y especialistas de la agencia para identificar la relación que existente entre necesidades de los clientes y servicios ofrecidos por la agencia.

Fase II: Evaluar el actual nivel de servicio: En esta fase se establecen los niveles de servicio y de calidad con los que opera la agencia. Esta, parte de un criterio clave: aumentar la satisfacción del cliente y superar sus expectativas. Para la evaluación de esta segunda fase se aplicó el cuestionario de evaluación autoevaluación del nivel de servicio, definido por Ulacia, (2015), el cual permitió medir las dimensiones relacional y operacional del servicio. El cuestionario fue aplicado al grupo de especialista de la agencia, antes mencionado, y a la dirección comercial, utilizando el mismo, como un método de auto-evaluación de la agencia, además fue desarrollado, por la autora de la presente investigación, como un método de evaluación, promediando las puntuaciones de ambas dimensiones para graficarlas en la matriz.

Según Fisher (1994) la dimensión operacional o de procedimiento es la que aporta la organización necesaria para lograr la consistencia, la eficacia y la eficiencia del servicio. La existencia de esta dimensión, provoca un servicio muy técnico, pero poco amable. Es un estilo de servicio que no admite flexibilidad, por lo que no refleja una buena actitud ante el servicio. Como ejemplos de esta dimensión se pueden identificar los siguientes aspectos: el local, la tecnología, el equipamiento, procesos y capacidad, comunicación y sistemas de información, y fuerza de trabajo.

Por el contrario, la dimensión relacional o de actitudes persigue construir un equilibrio entre las dos dimensiones, y, al mismo tiempo aporta la flexibilidad necesaria para los casos que 
se requieran hacer modificaciones en la dimensión operacional, con el fin de adaptar los procedimientos a las cambiantes exigencias del servicio y del cliente.

Fase III: Determinar los aspectos a mejorar: Con el fin de alcanzar los estándares fijados, se hace necesario evaluar el actual nivel de servicio y así poder identificar cuáles son los aspectos a mejorar y cuáles deberán ser modificados. Para la realización de este paso se aplicaron encuestas a los clientes externos, además se aplicó la ley de Pareto, conocida también como la Ley 80/20, la cual muestra que, para diversos casos, el $80 \%$ de las consecuencias proviene del $20 \%$ de las causas. El diagrama de Pareto, posibilitó la determinación de los principales aspectos a mejorar y consiste en un gráfico de barras, que clasifica de izquierda a derecha en orden descendente las causas o factores detectados en torno a un fenómeno. Finalmente se estableció el diagrama de afinidad, el cual es una herramienta usada, una vez aplicada la tormenta de ideas; el mismo permitió agrupar los factores, en grupos diferentes, que dan paso al problema planteado. A pesar de que esta metodología cuenta con 7 fases, la investigación se centrará en los tres primeros de la misma, ya que son los que dan cumplimiento al objetivo de la investigación. El resto de las fases que no se utilizaron en la presente investigación son el desarrollo de la estrategia, la implementación de la estrategia, la evaluación y la mejora continua.

\section{Principales resultados}

La agencia de viajes Gaviota Tours, es reconocida actualmente como líder en el mercado por poseer la mayor dinámica de crecimiento en el sector. Tiene más de 20 años de trabajo en conjunto con importantes operadores turísticos, y es la única agencia receptiva en Cuba reconocida con la Certificación del Sistema de Calidad ISO 9001, del 2008.

$\mathrm{Su}$ principal fortaleza es contar con la infraestructura del Grupo de Turismo Gaviota S.A, lo que le permite ofrecer productos a la medida, precios competitivos y un producto de mayor calidad, donde cada una de las partes agrega valor y está comprometida con todos los clientes de la compañía.

Gaviota Tours S.A está presente en los principales destinos turísticos del país, cuenta con la Sucursal Ventas Internacionales, la cual centraliza, de manera progresiva, la comercialización y contratación de sus servicios en los mercados, especializándose en el diseño de Circuitos, Paquetes Combinados, Eventos e Incentivos, Programas Especializados de Salud, Naturaleza y Servicios Premium.

Dentro de los principales mercados que adquieren algunos de los servicios anteriores, figuran Alemania y Francia, por ser estos los que mayor número de visitantes atraen y, además, los que mayores ingresos le generan a la agencia desde el año 2015.

La gestión de los servicios en la agencia de viajes Gaviota se ve afectada en varios puntos fundamentales, entre los que se destacan la falta de una metodología que responda al proceso 
de gestión de los servicios por parte de la entidad y la existencia de brechas en el servicio y por varios factores externos (el tecnológico y la competencia) los cuáles le brindan a la organización una imagen desventajosa frente a los clientes. Vale destacar, que, a pesar de ello, muchos tours operadores manifiestan que para ellos es mejor trabajar con la agencia de viajes Gaviota Tours, puesto que por la integración vertical que ha desarrollado, es la agencia que les facilita todos los servicios que ellos pueden buscar en el país ya que les asegura transporte, alojamiento, asesoría y hasta la recreación y otros servicios turísticos.

El tour operador Aventoura Cubareisen comienza las operaciones con Cuba en 1997, incluyendo al destino Cuba, no solo como un destino de sol y playa, sino como una experiencia de viaje de aventura y naturaleza, cultura y recreación. Se especializa en pequeños grupos y viajes individuales ofreciéndole a sus clientes que los encuentros con la naturaleza y la cultura sea una experiencia inolvidable y especial.

El tour operador TourCom es una agencia con más de 11 años de experiencia en el destino Cuba. Ofrece programas creativos para grupos de incentivos y de intereses especiales, enfocándose principalmente en la confección de productos a la medida. Trabaja fundamentalmente no solo con el mercado emisor Alemania, sino con todos aquellos países de habla germana, y además de habla inglesa. Los principales productos/servicios que ofrece la agencia a este tour operador, es la venta de paquetes a la medida, el servicio de alojamiento y la renta de autos.

A modo de conclusión en la primera etapa, se evaluó a la agencia dentro de la matriz de actitud personal, donde se mostró posicionada en el cuadrante de Presionadora (Figura 1), con un total de 11 puntos en la orientación al cliente y de 21 punto en orientación a la empresa, lo que indica que la agencia tiene una orientación definida más hacia a la entidad que a los clientes. Los aspectos identificados a mejorar son:

- Flexibilidad de la agencia con los clientes.

- El personal debe tener la libertad de negociar con el cliente, siempre y cuando el servicio lo requiera.

- La agencia debe cumplir con todo lo prometido al cliente.

- Debe darse solución a la totalidad de las reclamaciones de los clientes. 
Figura 1: Matriz de actitud personal

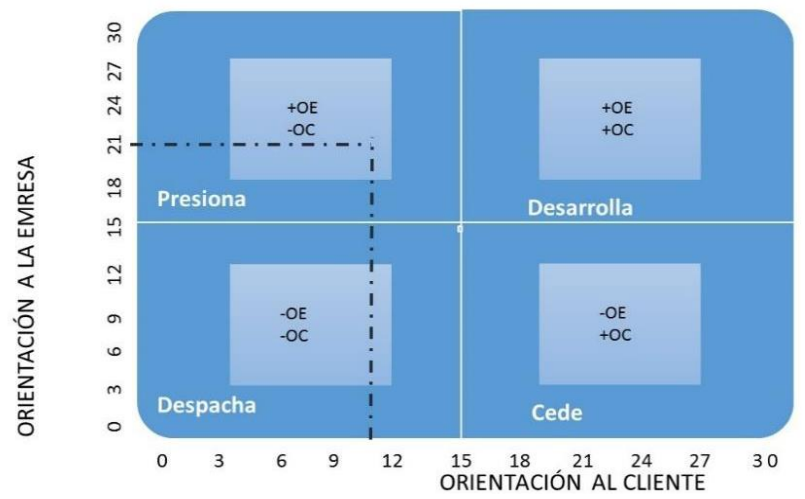

Fuente: Elaboración propia.

Se identificaron los momentos críticos de verdad, por los que atraviesan los tour operadores, determinándose como momento crítico de verdad de los ciclos de servicios en la agencia los siguientes:

1. Visita del tour operador a la agencia: Entra el tour operador a la agencia, Se entrevista con los especialistas, Valora contratos y la Salida del cliente.

2. Compra del programa: Solicitud del programa, Revisa la oferta, Recibe el servicio y Retroalimentación.

Se identificó la correspondencia existente entre los beneficios que ofrece la agencia y los atributos que percibe el cliente, mostrándose los principales problemas entre el servicio prometido y el servicio percibido (Ver figura 2).

Figura 2: Matriz de actitud personal

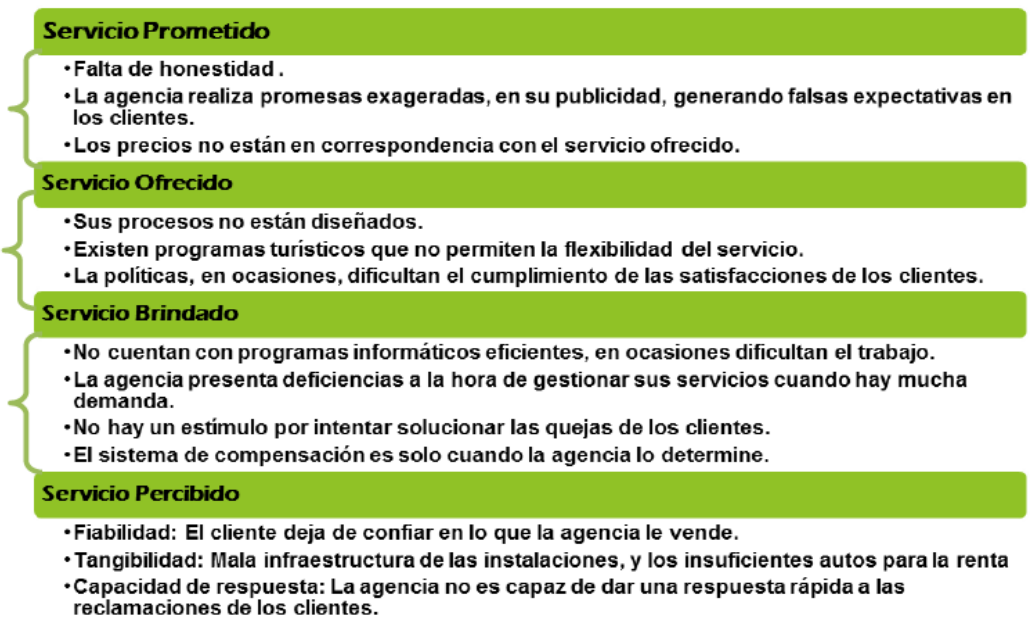

Fuente: Elaboración propia. 
Se creó la Matriz dimensional del servicio, a partir del análisis de las expectativas y necesidades que presenta el mercado y, en particular los clientes de la agencia y el desempeño del personal, y de la realización de una evaluación del servicio.

Una vez ubicado los puntos en la matriz se definió que el nivel de servicio que brinda la agencia está en el cuadrante mal servicio, lo que arroja consigo, una serie de aspectos a mejorar en las diferentes dimensiones, ya que son factores que no se llevan a cabo de manera adecuada, y están influyendo en la gestión de servicio de la agencia. Como ejemplo de estos factores que se definieron están:

1. Las políticas y procedimientos existentes en ocasiones obstaculizan la realización de un buen servicio.

2. La agencia no cuenta con un grupo de supervisores, que guíen, evalúen y controlen el trabajo realizado con sus clientes principales, como es el caso de los tour operadores alemanes Aventoura Cubareisen y TourCom, en materia de los servicios que ofrece la entidad.

3. Regularmente se mantienen problemas de servicios que han sido objetos de quejas de los clientes, por ejemplo: reclamaciones sobre la calidad del servicio de alojamiento, la no correspondencia entre la categoría vendida del hotel, y la que realmente se percibe, la poca disponibilidad de autos de renta, entre otros.

4. Ocasionalmente el personal da a conocer a los clientes, de manera indirecta, los problemas laborales.

5. No siempre el personal se preocupa de entregar un "extra" en su servicio al cliente.

6. No existe una flexibilidad de los servicios, con respecto a los requerimientos de los clientes.

7. No siempre, el personal promueve los servicios de la instalación, con técnicas efectivas.

8. No se estimula al personal.

A través del estudio realizado con el programa estadístico IBM SPSS Statistics, se pudo realizar un análisis acerca de las principales reclamaciones de los clientes por variables específicas y por servicios en general, obtenidos en la aplicación de las encuestas. En resumen, en la figura 3 se muestra el comportamiento de los diferentes servicios y las valoraciones de los clientes. 
Figura 3: Valoración de los clientes de los diferentes servicios.

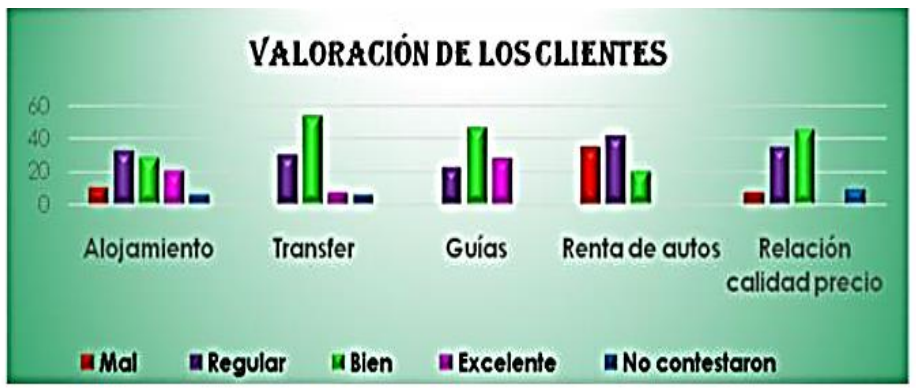

Fuente: Elaboración propia, a partir de los resultados de las encuestas aplicadas.

Posterior a ello, se aplicó el diagrama de Pareto (Ver figura 4), mediante el cual se determinó que la disminución de los ingresos por parte de dicho mercado estaba sustentada, fundamentalmente, en el elevado por ciento de insatisfacciones que reflejan los servicios de rentas de autos y el servicio de alojamiento, así como la percepción del cliente entre la calidad y el precio de los servicios ofertados. Vale destacar que los servicios fueron reordenados, según el por ciento de reclamaciones que presentaron.

Figura 4: Servicios a mejorar según la aplicación del Diagrama de Pareto

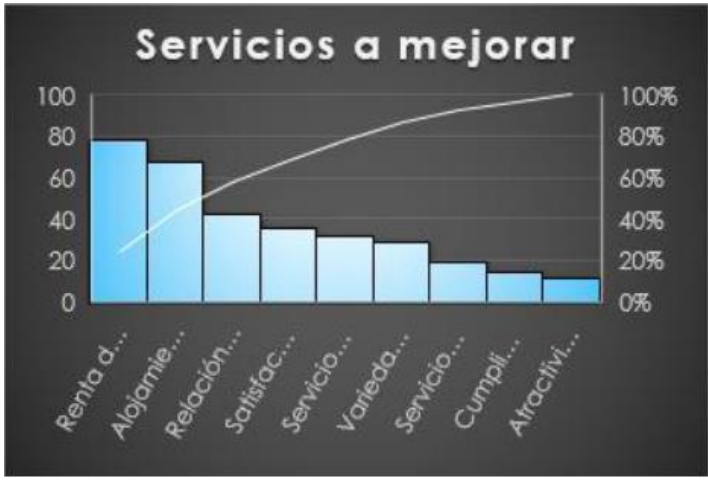

Fuente: Elaboración propia

Una vez determinado los principales servicios a mejorar, se realizó el Diagrama de Afinidad (Ver Figura 5), permitiendo agrupar las brechas identificadas y aspectos negativos que influían en dichos servicios. 
Figura 5: Diagrama de Afinidad. Factores que influyen en la disminución de los ingresos.

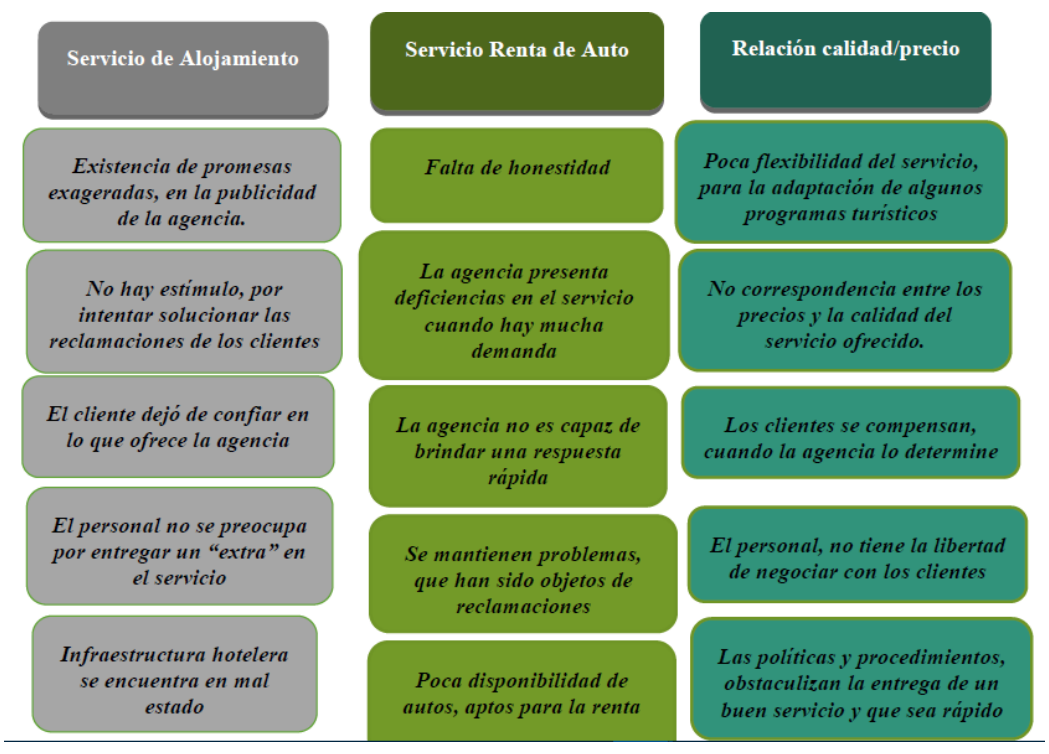

Fuente: Elaboración propia.

\section{Discusión}

La agencia de viajes Gaviota Tours S.A, posee grandes potencialidades para ejercer un desarrollo efectivo de los productos/servicios que brinda, al pertenecer al Grupo de Turismo Gaviota S.A, el cual presenta una integración vertical de sus servicios; por contar con entidades de alojamientos, transportistas, entidades de ocio y recreación, una agencia de viajes y una entidad proveedora, encargada de abastecer a las empresas mencionadas con anterioridad; estas entidades tienen como política priorizar el servicio solicitado por la agencia de viajes Gaviota Tours. Siendo esta, una de las ventajas que los tour operadores plantean que posee esta agencia, prefiriendo establecer contratos con ella.

Sin embargo, al identificar las brechas que existen entre los diferentes niveles del servicio a los clientes, sobresalieron, la incidencia en la gestión de la agencia, de algunos factores externos. Ejemplo de ellos:

\section{Factor tecnológico:}

- Insuficiente tecnología con la que cuenta la agencia: En el mundo, la tecnología avanza diariamente. Cuba, por su parte, se encuentra alejada de esta realidad. Aunque los tour operadores venden sus paquetes on-line, la agencia no cuenta con esa facilidad, la venta de sus servicios se realiza directamente en el país.

- Ineficiencia de algunos programas informáticos utilizados en la agencia: los programas informáticos con los que se trabaja, presentan dificultades, demorando en ocasiones, la entrega de los servicios a los clientes. 


\section{Competencia:}

- Pérdida de algunos posibles contratos: Los tour operadores mencionados con anterioridad, tienen que establecer contratos con otras agencias, dado que Gaviota Tours no puede brindarles algunos de los servicios demandados.

- Diferencia de precios: El tour operador, compara precios en todo momento, relacionándolo con la calidad de los servicios que se les ofrece, eligiendo, servicios brindados por otras agencias.

$\mathrm{Al}$ quedar identificados los factores y las brechas (Anexo 1) que inciden en la disminución de los ingresos, se elaboró un diagrama de Pareto (Figura 6), el cual permitió determinar los aspectos fundamentales que influyen en la disminución de los ingresos por parte del mercado alemán.

Figura 6: Diagrama de Pareto, para determinar los factores y brechas fundamentales.

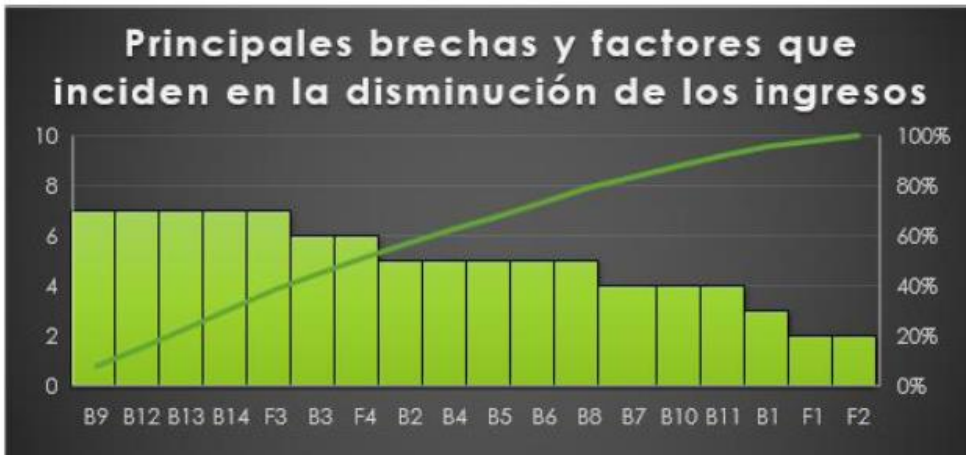

Fuente: Elaboración propia.

El análisis arrojó como resultado final, que los principales factores y brechas que inciden en la disminución de los ingresos por parte del mercado alemán son:

- Falta de estímulo hacia los trabajadores, para la solución de quejas de los clientes.

- Deterioro en las infraestructuras hoteleras.

- Falta de respuesta rápida, por parte de la agencia, a las reclamaciones de los tour operadores.

- Insuficiente número de autos, aptos para la renta.

- Pérdida de algunos posibles contratos con los tour operadores.

- Falta de correspondencia entre la calidad y el precio de los servicios ofrecidos.

- Diferencia de precios con relación a la competencia.

A raíz del estudio realizado, se definen a continuación, un conjunto de acciones, que respondan a la mejora de los principales problemas identificados, con el objetivo de disminuir 
las insatisfacciones en los clientes, mejorar la entrega de los servicios que más dificultades presentan, así como aumentar los ingresos de la agencia, con respecto al mercado alemán.

- Realizar contrataciones con empresas informáticas, para garantizar la existencia de programas y equipos tecnológicos, que faciliten la entrega de los servicios de la entidad.

- Realizar, mensual o trimestralmente, un estudio del comportamiento del mercado, en términos de ingresos y niveles de satisfacciones en los tours operadores.

- Diseñar los diferentes procesos de la entidad, para que sirvan como guía en la realización y el control del trabajo.

- Adaptar los programas existentes a los requerimientos y necesidades de los clientes.

- Diseñar nuevos productos/servicios específicos para cada mercado, según las características de sus clientes.

- Adaptar los programas existentes a los requerimientos y necesidades de los clientes.

- Diseñar nuevos productos/servicios específicos para cada mercado, según las características de sus clientes.

- Capacitar a un grupo de supervisores para que evalúen la entrega de los servicios brindados por los diferentes prestatarios.

- Establecer un plazo máximo a los proveedores para responder a las reclamaciones de los clientes.

- Diseñar un sistema de compensación para los clientes.

- Desarrollar un programa de estímulos para los trabajadores de la agencia.

- Establecer contratos con una mayor cantidad de arrendatarios particulares.

- Realizar un estudio de costos para cada uno de los productos/servicios ofertados.

- Estudiar periódicamente el comportamiento de la competencia.

\section{Conclusiones}

- La revisión bibliográfica realizada permitió conocer y comprender las particularidades de la gestión de los servicios en el sector turístico, en especial en las agencias de viajes.

- El diseño de una metodología, que responda a la gestión de los servicios, permitió el estudio del comportamiento de la misma por parte de la agencia con los tour operadores alemanes Aventoura Cubareisen y TourCom.

- Se determinaron las brechas y los factores fundamentales, que influían en la disminución de los ingresos por parte del mercado alemán.

- La disminución de los ingresos es consecuencia del nivel de insatisfacción que presentaron los clientes con respecto a los servicios de alojamiento, renta de autos, y la percepción entre la calidad y el precio. 


\section{Referencia bibliográfica}

Armenteros, E.D y Pelegrín, N (2011). Procedimientos para la elaboración del Plan estratégico de la Agencia de Viajes UEB Cubatur Polo Centro. Tesis de maestría. Facultad de Ingeniería Industrial y Turismo. Universidad Central Marta Abreu de las Villas. Trinidad. Cuba.

Benavides, L.H., y Guajala, M.M. (2011). Gestión, Liderazgo y valores en la administración de la unidad educativa "San Juan de Bucay" del Cantón. General Antonio Elizalde. Durante período 2010-2011. Tesis de maestría. Ciencias de la educación. Guayaquil.

Carlzon, J. (1991). El momento de la verdad. España: Díaz de Santos.

Castaño, Y. y Castillo, M. (2015). La promoción Turística a través de técnicas tradicionales y nuevas. Una revisión de 2009-2014. Universidad de Medillín. Colombia.

Colectivo de Autores. (2013). Importancia de las Agencias de Viajes. ARQYS. En http://www.arqys.con/contenidos/viajesagencas-importancia.html. Consultado: 10.02.2018

Fernández, C. y Blanco, A. (1996). Producción y Venta de Servicios Turísticos en Agencias de Viajes. España : Síntesis.

Fisher, R. (1994). El desafío del servicio. Material del seminario. Chile.

Saldaña, M.L. (2013). Estructura y Administración de Agencias de Viajes. Estado de México: Red Tercer Milenio, S.C.

Ulacia, Z. (2007). La gestión de Procesos en la Hospitalidad. Universidad de La Habana.

Ulacia, Z. (2015). Sistemas de Servicio y Hospitalidad. La Habana: Félix Varela.

Villamayor, C. y Lamas, E. (1998). Gestión de la radio comunitaria y ciudadana. Quito: MMRC

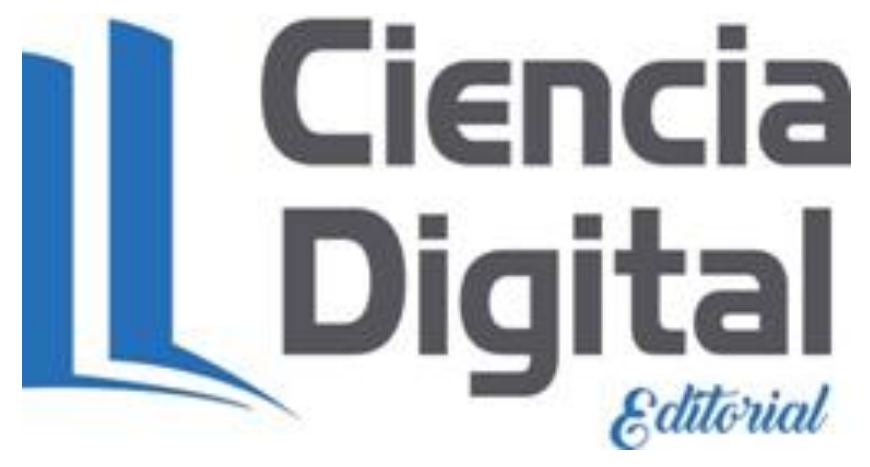




\section{Para citar el artículo indexado.}

Rodríguez M., Viamonte C., Hernández Y., Sánchez Y. \& Velasteguí E., (2019) La gestión de servicios de la Sucursal Ventas Internacionales de la agencia de viajes Gaviota Tours con los tour operadores alemanes Aventoura Cubareisen y TourCom. Revista electrónica Ciencia Digital 3(1), 394-409. Recuperado desde:

http://cienciadigital.org/revistacienciadigital2/index.php/CienciaDigital/article/view/309/72 $\underline{1}$

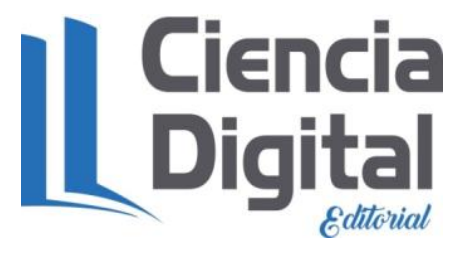

El artículo que se publica es de exclusiva responsabilidad de los autores y no necesariamente reflejan el pensamiento de la Revista Ciencia Digital.

El articulo queda en propiedad de la revista y, por tanto, su publicación parcial y/o total en otro medio tiene que ser autorizado por el director de la Revista Ciencia Digital.
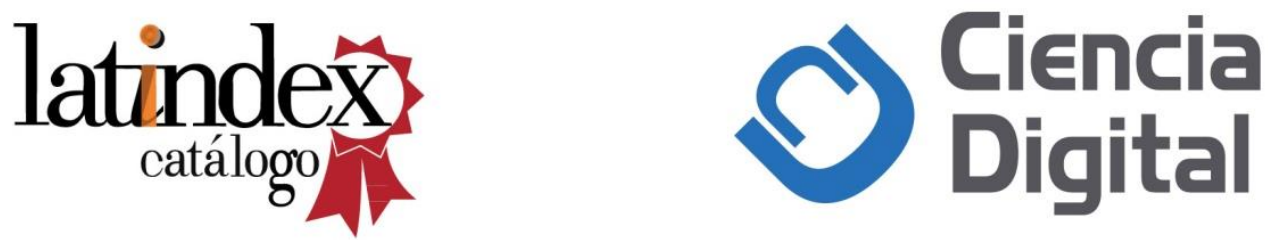